\title{
MECHANICAL SUPPORT WITH MICROAXIAL BLOOD PUMPS FOR POSTCARDIOTOMY LEFT VENTRICULAR FAILURE: CAN OUTCOME BE PREDICTED?
}

Bart Meyns, MD, $\mathrm{PhD}^{\mathrm{a}}$

Paul Sergeant, MD, $\mathrm{PhD}^{\mathrm{a}}$

Patrick Wouters, $\mathrm{MD}, \mathrm{PhD}^{\mathrm{b}}$

Filip Casselman, MD

Paul Herijgers, $\mathrm{MD}, \mathrm{PhD}^{\mathrm{a}}$

Willem Daenen, MD

Kris Bogaerts ${ }^{c}$

Willem Flameng, $\mathrm{MD}, \mathrm{PhD}^{\mathrm{a}}$
Objective: We sought to identify the indications of mechanical support in postcardiotomy left ventricular failure in patients who are unable to undergo transplantation.

Methods: From 1989 through 1997, 61 patients with postcardiotomy left ventricular failure beyond intra-aortic balloon pumping were assisted with the Hemopump cardiac assist system (Medtronic, Minneapolis, Minn). Their mean age was $64 \pm 8$ years. Comorbidity was prevalent; $47 \%$ underwent cardiac massage before pump support, and evolving myocardial infarction was diagnosed in $43 \%$ before surgery. Multivariable logistic regression of data known at the moment of pump insertion was performed to identify the risk factors for mortality.

Results: Sixty-five percent of the patients were weaned from the device, but only 30\% were discharged home. Cardiac index evolution during the first hours after pump insertion $(P<.001)$ is the only independent predictor for possibility to wean from the device in the multivariable analysis. Acute renal failure is the only variable retained in the model for 90-day mortality. Device-related complications were far more frequent with the femoral (54\%) than with the transthoracic $(6 \%)$ cannula. Only $13 \%$ of the patients had bleeding complications.

Conclusions: One third of the patients with postcardiotomy heart failure refractory to use of the intra-aortic balloon pump can be saved with the use of an endovascular axial flow pump. It is impossible to predict lethal outcome on preoperative data alone. The early hemodynamic response to support seems to be related to functional recovery of the heart and subsequent weaning from the device. (J Thorac Cardiovasc Surg 2000;120:393-400)
P ostcardiotomy heart failure is the most troublesome condition in cardiac surgery. Patients unable to be weaned or patients undergoing failure in the first hours after surgery are generally treated with increased inotropic support and use of an intra-aortic balloon

From the Department of Cardiac Surgery, ${ }^{\mathrm{a}}$ the Department of Cardiac Anesthesiology, ${ }^{\mathrm{b}}$ and the Biostatistical Center, ${ }^{\mathrm{c}} \mathrm{KU}$ Leuven, Belgium.

Received for publication July 16, 1999; revisions requested Sept 13, 1999; revisions received April 12, 2000; accepted for publication April 12, 2000.

Address for reprints: B. Meyns, MD, PhD, Department of Cardiac Surgery, UZ Gasthuisberg, Herestraat 49, 3000 Leuven, Belgium (E-mail: bart.meyns@uz.kuleuven.ac.be).

Copyright (C) 2000 by The American Association for Thoracic Surgery

0022-5223/2000\$12.00+ $0 \quad \mathbf{1 2 / 1 / 1 0 7 8 3 3}$

doi: $10.1067 / \mathrm{mtc} .2000 .107833$ pump (IABP). Their survival to hospital discharge is reported to be around 50\% ${ }^{1,2}$ At this moment, it is still unclear whether patients with heart failure should be treated immediately with more powerful assist devices and in which patients further therapy is useless. Since 1989 we used, in a consecutive series of patients with postcardiotomy left ventricular failure, the Hemopump cardiac assist system (Medtronic, Minneapolis, Minn) as the first-choice assist device in patients unsuitable for transplantation because of their age or comorbidity. We analyzed our data to delineate the indications for mechanical support in heart failure.

\section{Methods}

Left ventricular failure strategy. If a patient cannot be weaned from extracorporeal circulation, reperfusion is allowed for half an hour. In that period, an IABP is placed and 


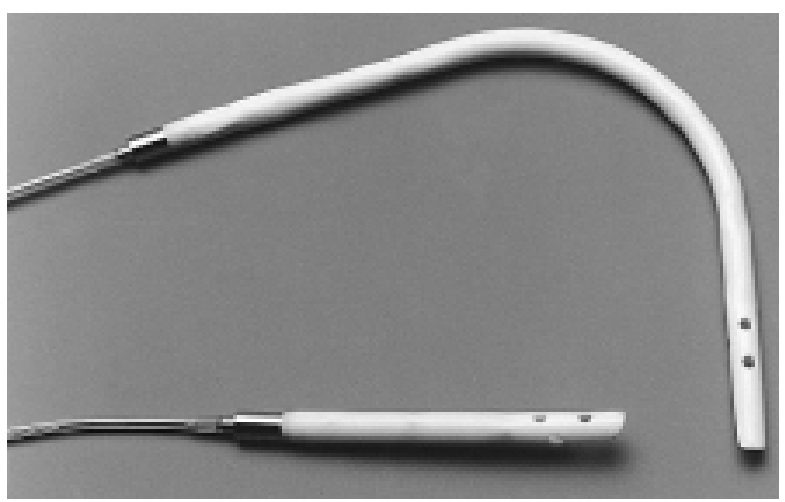

Fig 1. The Hemopump cardiac assist system is a miniaturized axial rotary blood pump. There were two cannulas in clinical use: the long femoral type and the short transthoracic type. Both are $8.1 \mathrm{~mm}$ wide.

medical therapy is optimized. Medical therapy comprises optimal filling; inotropic support with dobutamine, dopamine, and enoximone; and, in cases of vasodilation, norepinephrine. A second weaning attempt is undertaken. If signs of left ventricular failure are still obvious (ie, cardiac index $<2 \mathrm{~L} \cdot \mathrm{m}^{-2} \cdot \mathrm{min}^{-1}$ or systolic blood pressure $<90 \mathrm{~mm}$ $\mathrm{Hg}$ with increased left atrial pressures), the Hemopump device is inserted.

In cases in which left ventricular failure becomes obvious some hours after surgery in the intensive care unit, medical therapy is optimized, and an IAPB is inserted as a measure of intensive care. If hemodynamics again indicate a persistently failing ventricle, the patient is taken back to the operating room, and the Hemopump device is inserted.

The device. The Hemopump cardiac assist system is a miniaturized rotary blood pump mounted on a catheter (Fig 1). The rotor is driven by an electric motor. The bedsideinstalled console allows regulation of the pump speed from 17,000 to 26,000 rotations per minute. Once the cannula is placed over the aortic valve, it sucks the blood out of the left ventricle and expells it into the ascending aorta. Two cannulas were used in this series: a femoral device and a transthoracic device. Both are 8.1-mm wide, but the femoral cannula has a longer inflow tip and is less performant (maximum flow of $3.5 \mathrm{~L} / \mathrm{min}$ for the femoral and $5.1 \mathrm{~L} / \mathrm{min}$ for the transthoracic cannula). The nonpulsatile flow delivered by the Hemopump device is continuously influenced by the underlying cardiac activity. ${ }^{3,4}$ The Hemopump console does not display the actually delivered flow, but a performance indicator shows the varying motor current. From 1989 through 1992, the femoral cannula was used. From the first of January 1993, we switched to the transthoracic cannula.

Patients. From 1989, the date of introduction of the Hemopump device in our institution, through December 31, 1997, 12,163 patients underwent cardiac surgery. Three hundred eighty-eight (3.2\%) patients received an IABP in the perioperative period, and $108(0.9 \%)$ patients were sup- ported with a more powerful assist device. If bridging to heart transplantation was the first option, the patient was assisted with a medium-term pulsatile assist device (either Abiomed from Abiomed Cardiovascular, Inc, Danvers, Mass, or Medos from Medos, Stollberg, Germany). The Hemopump is a short-term assist device and was only considered in cases of isolated left ventricular failure, with the intention of recovering the cardiac function. Patients with clinical signs of right ventricular or biventricular failure received a right or biventricular assist device other than the Hemopump device (centrifugal pump, Abiomed, and Medos). The assessment of right ventricular function was based on hemodynamic status (central venous pressure, pulmonary artery pressure, and cardiac output), on echocardiographic assessment of the right ventricular contractility, and on clinical background. Therefore, 61 patients received the Hemopump system for isolated left ventricular failure in a postcardiotomy setting, with recovery of the heart as the only possible outcome.

Table I summarizes the biographical data of these 61 patients with a mean age of $64 \pm 7.9$ years. Many of them were operated on under emergency conditions. Almost half of these patients $(47 \%)$ underwent cardiopulmonary resuscitation before the Hemopump insertion, and in $43 \%$ of them, the diagnosis of evolving myocardial infarction was known at the moment of Hemopump initiation.

Important comorbidity is present with predominantly vascular pathology and diabetes. The comorbidity and age were exclusion criteria for heart transplantation for each of these patients.

Surgical technique. The femoral Hemopump device is inserted through a cutdown of the femoral artery. Bleeding on insertion is controled by means of a $12-\mathrm{mm}$ Dacron graft sutured to the artery. The cannula is introduced through the graft and then passed across the aortic arch and the aortic valve under fluoroscopic guidance.

The transthoracic Hemopump device is inserted through a graft sutured onto the ascending aorta. The cannula is passed over the aortic valve under direct palpation of the ascending aorta. The position of the cannula is confirmed by means of transthoracic echocardiography. Occluding plugs around the drive cable prevent blood loss through the graft once the cannula is in place. Tying one plug close to the ascending aorta secures the position of the cannula and prevents displacement.

Hemopump assist systems are inserted without extracorporeal circulation, with the exception of the patients unweanable from bypass. The transthoracic drive cable is positioned with a smooth curve in the right pleural space and leaves the chest through the caudal end of the sternotomy.

Removal of the transthoracic device requires resternotomy. The graft is occluded as low as possible and sutured.

Patient management. Inotropic support is stopped as soon as possible. Vasopressors are continued in cases of vasodilation. Hemodynamic goals are a mean aortic blood pressure of $70 \mathrm{~mm} \mathrm{Hg}$, with left atrial and right atrial pressures of 10 to $15 \mathrm{~mm} \mathrm{Hg}$. Filling volume is primarily triggered by the right 
Table I. Biographic data of the patients supported with the Hemopump device $(n=61)$

\begin{tabular}{|c|c|c|c|c|}
\hline & No. & Observations & $\%$ & Mean $\pm S D$ \\
\hline Age (y) & 61 & & & $64 \pm 8.0$ \\
\hline Male sex & 61 & 45 & 74 & \\
\hline CPR before assist device & 61 & 29 & 47 & \\
\hline Acute myocardial infarction & 60 & 27 & 45 & \\
\hline Left ventricular hypertrophy & 59 & 19 & 32 & \\
\hline Acute renal failure & 60 & 20 & 33 & \\
\hline Peripheral vascular disease & 59 & 16 & 27 & \\
\hline Diabetes & 59 & 13 & 22 & \\
\hline Chronic obstructive pulmonary disease & 60 & 4 & 7 & \\
\hline Chronic renal failure & 60 & 3 & 5 & \\
\hline Duration of shock (h) & 58 & Median, 1 & $(25 \%-75 \%: 0.25-2.3)$ & \\
\hline Weight $(\mathrm{kg})$ & 52 & & & $73 \pm 0.8$ \\
\hline Length (cm) & 52 & & & $169 \pm 0.4$ \\
\hline Cardiac index before assist device $\left(\mathrm{L} \cdot \mathrm{m}^{-2} \cdot \mathrm{min}^{-1}\right)$ & 39 & & & $1.8 \pm 0.8$ \\
\hline Mean blood pressure before assist device $(\mathrm{mm} \mathrm{Hg}$ ) & 41 & & & $54 \pm 12$ \\
\hline Left atrial pressure before assist device $(\mathrm{mm} \mathrm{Hg})$ & 35 & & & $19 \pm 5$ \\
\hline Cardiac index after assist device $\left(\mathrm{L} \cdot \mathrm{m}^{-2} \cdot \mathrm{min}^{-1}\right)$ & 47 & & & $3.3 \pm 0.9$ \\
\hline Mean blood pressure after assist device ( $\mathrm{mm} \mathrm{Hg}$ ) & 53 & & & $7.5 \pm 4$ \\
\hline Left atrial pressure after assist device $(\mathrm{mm} \mathrm{Hg})$ & 51 & & & $10 \pm 3$ \\
\hline
\end{tabular}

$C P R$, Cardiopulmonary resuscitation.

atrial pressure because the right ventricular function is considered to be the limiting factor. Cardiac output by the thermodilution method indicates right ventricular output and reflects the sum of both Hemopump and left ventricular output. Besides the hemodynamic status and clinical signs, plasma lactate levels are monitored to estimate peripheral organ perfusion. In cases in which an IABP is present, we leave it in place because we assume a beneficial effect to myocardial blood flow. ${ }^{5}$

All patients remain sedated and mechanically ventilated during Hemopump support.

Once the drainage through the chest tubes indicates a dry surgical field, we start a continuous heparin infusion to obtain activated clotting time between 180 and 200 seconds.

The antibiotic routine consists of $3 \times 2 \mathrm{~g}$ of a second-generation cephalosporin the day of surgery. Additional antibiotic therapy is only initiated on guidance of clinical signs of infection.

When recovery of the heart becomes obvious (by increasing pulsatility of the arterial blood pressure), we wean the patient gradually over several days by reducing the pump speed. Transesophageal echocardiography is performed on indication (like clinical suspicion of cardiac tamponade). On the last day, with minimal Hemopump support, transesophageal echocardiography is performed to judge myocardial contractility and to plan the pump removal.

Statistical analysis. Continuous data are presented as means and SD. Paired data are analyzed with the paired Student $t$ test. A Kaplan-Meier life-table curve was constructed with the statistical software package Statistica (StatSoft Inc, Tulsa, Okla). Because mortality occurred in a rather short period of time, a logistic regression analysis was performed to predict 90-day mortality.
Simple logistic regression and multivariable logistic regression (SAS; SAS Institute Inc, Cary, NC) were performed to analyze the influence of different clinical variables on the possibility of weaning the patients from the pump and on 90-day mortality. The following variables known by the surgeon at the time of urgent surgery, existing largely of crude historical and hemodynamic data, were considered: age; weight; length; the need for cardiopulmonary rescucitation before surgery; the duration of shock before surgery (ie, shock is a cardiac index $<2 \mathrm{~L} \cdot \mathrm{m}^{-2} \cdot \mathrm{min}^{-1}$ or systolic pressure $<90 \mathrm{~mm} \mathrm{Hg}$ with elevated filling pressures); cardiac index before surgery; mean blood pressure before surgery; left atrial pressure before surgery; peripheral vascular disease; diabetes; chronic renal failure (creatinine $>2.5 \mathrm{mg} / \mathrm{dL}$ or hemodialysis); acute renal failure (no urine output in the last hour); chronic obstructive lung disease; redo surgery; left ventricular hypertrophy; known severe reduction of ventricular ejection fraction; and evolving acute myocardial infarction at the moment of surgery. Furthermore, additional information gained by the immediate hemodynamic response to the pump insertion were also considered. These variables are cardiac index in the first 6 hours after insertion of the device, mean arterial blood pressure in the first 6 hours after insertion of the device, and left atrial pressure in the first 6 hours after insertion of the device. The variables for which more than $25 \%$ of the data were missing were not allowed into the multivariable model.

\section{Results}

Weaning from the device. Immediate hemodynamic recovery was obvious, with a significant increase in cardiac index (from $1.8 \pm 0.7$ to $3.3 \pm 0.8 \mathrm{~L} \cdot \mathrm{m}^{-2}$. $\left.\min ^{-1} ; P<.0001\right)$ and mean arterial blood pressure 


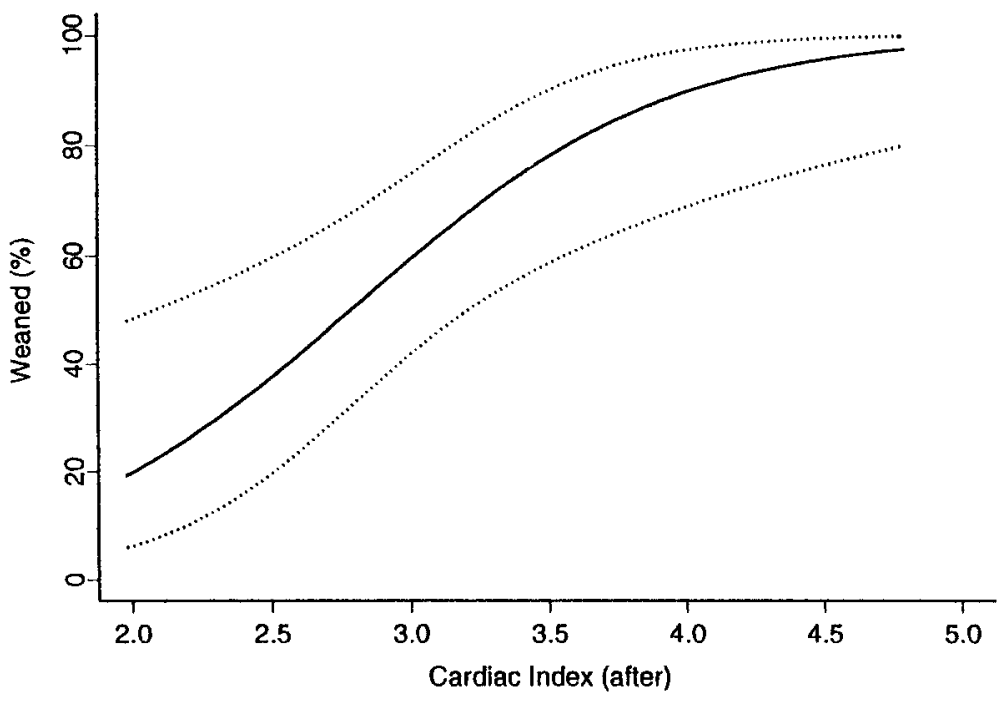

Fig 2. The relation (with 95\% confidence limits) between cardiac index in the first 6 hours after Hemopump support and the possibility of weaning the patient from the device.

Table II. Simple and multivariable logistic regression of risk factors indicating the possibility of weaning the patient from the device

\begin{tabular}{|c|c|c|c|}
\hline & $O R$ & $95 \% C I$ & $\mathrm{P}$ value \\
\hline \multicolumn{4}{|l|}{ Univariate analysis weaning } \\
\hline Sex (female vs male) & 1.336 & $0.396-4.510$ & $>.3$ \\
\hline Age & 0.952 & $0.884-1.025$ & .17 \\
\hline Weight & 0.924 & 0.855-0.999 & .04 \\
\hline Length & 0.969 & 0.894-1.049 & $>.3$ \\
\hline CPR & 1.111 & $0.389-3.177$ & $>.3$ \\
\hline Duration of shock & 1.012 & $0.954-1.074$ & $>.3$ \\
\hline Cardiac index ${ }^{*}$ & 0.966 & $0.396-2.353$ & $>.3$ \\
\hline Arterial blood pressure ${ }^{*}$ & 1.023 & $0.968-1.081$ & $>.3$ \\
\hline Left atrial pressure* & 1.000 & $0.860-1.163$ & $>.3$ \\
\hline Peripheral vascular disease & 1.179 & $0.345-4.029$ & $>.3$ \\
\hline Diabetes & 0.510 & $0.145-1.796$ & .30 \\
\hline Chronic renal failure & 0.000 & $0.000-1.244$ & .07 \\
\hline Acute renal failure & 0.135 & $0.040-0.448$ & $<.001$ \\
\hline Chronic pulmonary disease & 1.667 & $0.162-17.100$ & $>.3$ \\
\hline Redo surgery & 1.120 & $0.366-3.428$ & $>.3$ \\
\hline Left ventricular hypertrophy & 0.54 & $0.172-1.701$ & .29 \\
\hline Poor ejection fraction & 0.583 & $0.189-1.803$ & $>.3$ \\
\hline Acute myocardial infarction & 0.85 & $0.293-2.465$ & $>.3$ \\
\hline Cardiac index (after) & 5.861 & $1.881-18.264$ & $<.001$ \\
\hline Mean blood pressure (after) & 1.056 & $1.004-1.110$ & .02 \\
\hline Left atrial pressure (after) & 0.982 & $0.817-1.181$ & $>.3$ \\
\hline \multicolumn{4}{|l|}{ Multivariable model weaning } \\
\hline Cardiac index (after) & 5.861 & $1.881-18.264$ & $<.001$ \\
\hline
\end{tabular}

$O R$, Odds ratio; $C I$, confidence interval; $C P R$, cardiopulmonary resuscitation before insertion of the device; after, hemodynamic values obtained in the first 6 hours after insertion of the device.

*Variable that is excluded from the multiple model because greater than $25 \%$ of the data are missing.

(from $54 \pm 12$ to $74 \pm 14 \mathrm{~mm} \mathrm{Hg} ; P<.0001$ ) and a decrease in left atrial pressure (from $19 \pm 5$ to $10 \pm 3$ $\mathrm{mm} \mathrm{Hg} ; P<.0001)$. Weaning from the device was possible in $39(65 \%)$ of the patients. Weaning was intend- ed within the first week (longest duration on the device was 10 days). Twenty percent of the patients died the very first day of support. In all these cases a technical pump failure was excluded. Mode of death in these 


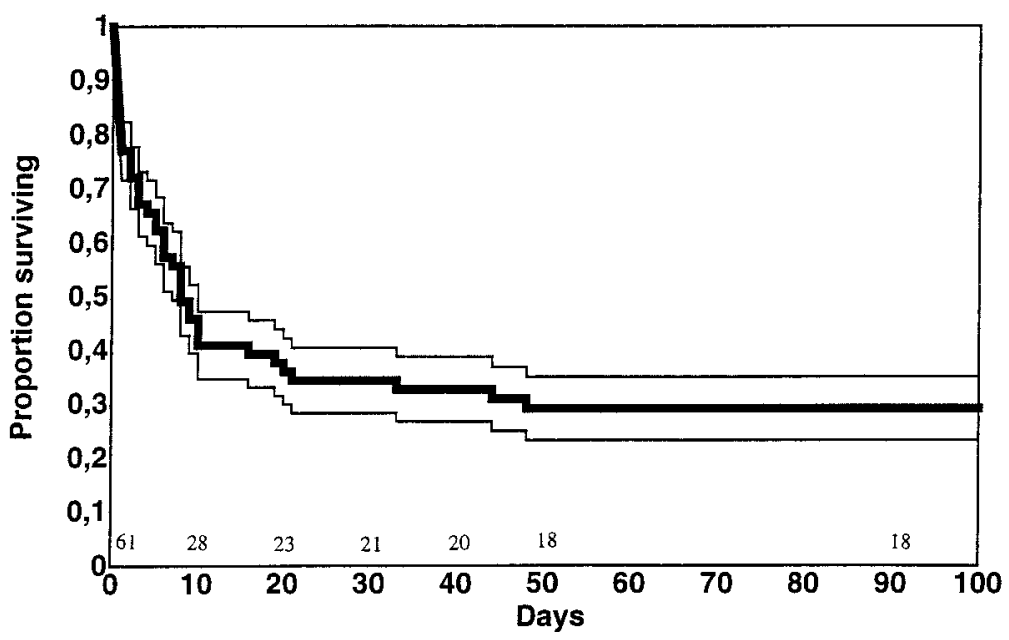

Fig 3. Kaplan-Meier survival curve of 61 patients with postcardiotomy heart failure supported by the Hemopump device; the number of patients at risk is indicated above the horizontal axis. The assist device was in all cases removed before day 10 . Weaning was possible in $65 \%$ of the patients, and only $30 \%$ were discharged home. One patient died after discharge.

early failures was progressive vasodilation resistant to high-dose vasopressors and insufficient organ perfusion or discontinuation of therapy because of evidence of brain death. Table II shows the clinical data known in these emergency conditions and the level of their significance to predict the possibility to wean the patient from the device.

Body weight, acute renal failure, cardiac index, and mean aortic blood pressure after the pump is started are significant risk factors in a univariate analysis (Table II). Of the 20 patients in acute renal failure (defined as no urine output in the last hour), 6 had an immediate improvement of the renal function (normal urine output) once the mechanical support was initiated. Of these 6 patients, 5 could be weaned from the device. On the other hand, only 2 of the 14 patients without recuperation of the renal function could be weaned.

Once the device is inserted, additional information is derived from the immediate hemodynamic response. Cardiac index and mean arterial blood pressure after use of the pump are significant predictors. However, the multivariable logistic regression only retains cardiac index after support as a significant factor. Fig 2 shows the relationship between cardiac index and the possibility to wean the patient from the device. In our series no patient with a cardiac index of less than $2.5 \mathrm{~L} \cdot \mathrm{m}^{-2} \cdot \mathrm{min}^{-1}$ in the first hours after insertion of the Hemopump device survived to be weaned.

Survival. The Kaplan-Meier survival curve (Fig 3) indicates the drop in survival during the first week before the patients were weaned. Although $65 \%$ of the patients were weaned, only $30 \%$ were discharged home alive. One patient died after discharge (mean follow-up of 197 days). Death after weaning from the device was due to late consequences of organ failure (eg, respiratory failure, sepsis, and hepatic failure) and low cardiac output. Table III lists the clinical data known in the operating room and their level of significance to predict 90-day mortality. Only acute renal failure and mean arterial blood pressure after the support is commenced are significantly related to 90-day mortality in a univariate way. The mutivariable logistic regression retains only acute renal failure.

Device-related complications. There is a striking difference in occurrence of device-related complications between the earlier experiences with the femoral device and the later experiences with the transthoracic pump. Table IV summarizes the device-related complications. Problems of cannula introduction and drive cable fracture were only seen with the femoral device. Hemolysis (defined as plasma-free hemoglobin $>40$ $\mathrm{mg} / \mathrm{dL}$ ) was only present in cases of pump blockage by thrombus. Bleeding (defined as revision for bleeding or tamponade or blood loss exceeding $1500 \mathrm{~mL} / 12$ hours) occurred in $13 \%$ of the patients. We registered clinical signs of embolic phenomena in none of these patients. However, no specific investigation was performed to exclude the occurrence of emboli.

\section{Discussion}

In 1965, Spencer and colleagues ${ }^{6}$ reported the first successful use of ventricular support in patients with 
Table III. Simple and multivariable logistic regression of risk factors for 90-day mortality

\begin{tabular}{|c|c|c|c|}
\hline & $O R$ & $95 \% C I$ & $\mathrm{P}$ value \\
\hline \multicolumn{4}{|l|}{ Univariate analysis mortality } \\
\hline Sex (female vs male) & 0.606 & $0.181-2.030$ & $>.3$ \\
\hline Age & 1.067 & $0.994-1.145$ & .06 \\
\hline Weight & 1.072 & $0.995-1.155$ & .05 \\
\hline Length & 1.045 & $0.962-1.137$ & .29 \\
\hline $\mathrm{CPR}$ & 0.574 & $0.188-1.781$ & $>.3$ \\
\hline Duration of shock & 1.021 & $0.946-1.101$ & $>.3$ \\
\hline Cardiac index* & 0.756 & $0.294-1.944$ & $>.3$ \\
\hline Arterial blood pressure* & 0.954 & $0.898-1.014$ & .11 \\
\hline Left atrial pressure* & 1.138 & $0.963-1.344$ & .11 \\
\hline Peripheral vascular disease & 2.321 & $0.571-9.445$ & .22 \\
\hline Diabetes & 1.613 & $0.386-6.739$ & $>.3$ \\
\hline Chronic renal failure & $+\infty$ & $0.176-+\infty$ & .54 \\
\hline Acute renal failure & 6.000 & $1.221-29.480$ & .01 \\
\hline Chronic pulmonary disease & 1.308 & $0.127-13.491$ & $>.3$ \\
\hline Redo surgery & 0.786 & $0.250-2.468$ & $>.3$ \\
\hline Left ventricular hypertrophy & 1.4 & $0.414-4.737$ & $>.3$ \\
\hline Poor ejection fraction & 0.933 & $0.304-2.869$ & $>.3$ \\
\hline Acute myocardial infarction & 2 & $0.632-6.327$ & .23 \\
\hline Cardiac index (after) & 0.474 & $0.218-1.032$ & .05 \\
\hline Mean blood pressure (after) & 0.949 & $0.905-0.994$ & .02 \\
\hline Left atrial pressure (after) & 0.975 & $0.807-1.178$ & $>.3$ \\
\hline \multicolumn{4}{|l|}{ Multivariable model mortality } \\
\hline Acute renal failure & 6.000 & $1.221-29.480$ & .01 \\
\hline
\end{tabular}

$O R$, Odds ratio; $C I$, confidence interval; $C P R$, cardiopulmonary resuscitation before the insertion of the device; after, hemodynamic values obtained in the first 6 hours after insertion of the device; $\infty$, infinity.

*Variable that is excluded from the multiple model because greater than $25 \%$ of the data are missing.

Table IV. Device-related complications in 61 patients

\begin{tabular}{lcr}
\hline & $H P 21$ & $H P 31$ \\
\hline No. & 13 & 48 \\
Hours & 756 & 4455 \\
Cannula introduction (\%) & $3(23)$ & $0(0)$ \\
Cannula displacement (\%) & $4(31)$ & $1(2)$ \\
Thrombosed rotor (\%) & $1(8)$ & $2(4)$ \\
Drive cable fracture (\%) & $1(8)$ & $0(0)$ \\
Hemolysis (\%) & $3(23)$ & $2(4)$ \\
Intervention required (\%) & $7(54)$ & $3(6)$ \\
Bleeding (\%) & \multicolumn{2}{c}{$8(13)$} \\
Embolic phenomena (\%) & \multicolumn{2}{c}{$0(0)$} \\
\hline
\end{tabular}

Hemolysis is defined as a free plasma hemoglobin level of greater than 40 $\mathrm{mg} / \mathrm{dL}$. Bleeding is defined as revision for bleeding or tamponade or blood loss greater than $1500 \mathrm{~mL} / 12$ hours. HP21, Femoral cannula; HP31, transthoracic cannula.

postcardiotomy cardiac failure. Since then, the clinical applications of ventricular assist devices have grown, and several types of devices have been developed. Reports of the use of these devices to support postcardiotomy heart failure show a hospital survival of $20 \%$ to $30 \% .^{7-12}$ In earlier reports duration of support, older age, and the presence of acute renal failure were reported to be predictive for hospital mortality. ${ }^{8,12-14}$ However, later studies could not confirm these findings. ${ }^{10}$ Regardless, the type of device used, bleeding, and thromboembolic phenomena were the most frequent complications. ${ }^{9,12}$ Therefore, the implantation of an assist device remains a major surgical intervention not likely to be done on a prophylactic basis. In clinical practice an assist device is only used if all other options have failed. However, several studies showed that mechanical support reduces myocardial oxygen consumption and infarct size and enhances myocardial recovery after stunning. ${ }^{15-17}$ Exact knowledge of predictors for survival with and without assist devices is therefore crucial. Baldwin and colleagues ${ }^{2}$ identified complete heart block, female sex, advanced age, and preoperative blood urea nitrogen as predictive parameters for mortality in a group of 322 patients supported with an IABP. We accepted the common principle that whoever failed to do well with the IABP should receive a ventricular assist device. In our consecutive series of 61 patients treated with the same left ventricular assist device (the Hemopump assist system), we tried to identify the preoperative risk factors for survival. The factors that were shown to be significant 
were less related to the patients' general state but more an expression of the degree of organ failure that took place. The presence of acute renal failure is the only preoperative variable associated with 90-day mortality in the multivariable analysis. Second, the immediate hemodynamic recovery of the patient is significantly related to the possibility of weaning the patient. The cardiac index in the first hours after initiation of the device is the only significant predictor of the possibility to wean the patient from the device in the multivariable logistic regression. All patients with a cardiac index under $2.5 \mathrm{~L} \cdot \mathrm{m}^{-2} \cdot \mathrm{min}^{-1}$ within the first hours of support died in the first days. The inability to improve the hemodynamic state in these patients is probably based on the deep cardiogenic shock these patients had undergone before the pump was started. A general loss of vasotonus that is resistant to vasopressors is often the way this is expressed. The therapy in these patients was definitely insufficient or inappropriate. The information of the preoperative risk factors (acute renal failure) and the response to the initiation of mechanical support allows earlier decision making. This finding has brought us to define the clinical pathways of patients on an assist device in the operating room. If they do not meet the criteria of hemodynamic response within the first hours after the pump is placed, we decide immediately on further actions. This can lead to the use of biventricular support, the redirection of the patient as a bridge to transplantation, or the defiance of further invasive therapy. Not only the state of shock but also the myocardial possibility of recovery, as well as the general clinical state of the patient, are considered.

Although the analysis showed that the patients' degree of shock is the major predictor of survival, this only helps clinical decisions in patients who do not immediately respond to mechanical support. The known preoperative variables are or are related to the patient's general state (eg, age and vascular disease), the myocardial potential for recovery (eg, poor ejection fraction and acute myocardial infarction), or the degree of shock (eg, acute renal failure and hemodynamic data). Unfortunately, the overwhelming effect of the latter category interferes with all other variables in this specific patient group of postcardiotomy heart failure, with recovery as the only outcome. ${ }^{10}$ In this series as well, mechanical support was only initiated when all other therapy failed. This has led to a selection of patients with a high proportion of patients in clear shock (47\% were in cardiopulmonary resuscitation). The true value of the variables from the other categories will only be discovered when shock is less prevalent. This entails an earlier initiation of mechanical support. The earlier initiation of mechanical support, however, is only justifiable if the side effects of the device itself do not increase the risk for the patients. With this miniaturized microaxial blood pump, this evolution toward prophylactic use is possible.

We found an extremely low rate of bleeding complications $(13 \%)$. In our experience and that of others ${ }^{12}$ with other assist devices, the incidence of bleeding is reported to be as high as $50 \%$. Clearly, the simplicity of the device without the need for inflow and outflow cannulas is the underlying cause. The device proved to be simple to introduce and remove, with an acceptable thromboembolic complication rate and, as mentioned, an extremely low bleeding rate. Other groups have reported similar experiences with the Hemopump as an assist device. ${ }^{18-20}$ Unfortunately, this device is no longer available for clinical use. Several teams are working on the development of elegant rotary blood pumps. ${ }^{21-23}$ We look forward to this evolving technology and hope that the improved devices will also offer the same elegance and efficiency of the Hemopump system, such as increased durability. In that case the search for the ideal indications of the least invasive mechanical support can be continued.

\section{REFERENCES}

1. Arafa OE, Pedersen TH, Svennevig JL, Fosse E, Geiran O. Intraaortic balloon pump in open heart operations: 10-year follow-up with risk analysis. Ann Thorac Surg 1998;65:741-7.

2. Baldwin RT, Slogoff S, Noon GP, et al. A model to predict survival at time of postcardiotomy intraaortic balloon pump insertion. Ann Thorac Surg 1993;55:908-13.

3. Meyns B, Siess T, Laycock S, Reul H, Rau G, Flameng W. The heart-Hemopump interaction: a study of Hemopump flow in function of cardiac activity. Artif Organs 1996;20:641-9.

4. Siess T, Meyns B, Spielvogel K, Ruel H, Rau G, Flameng W. Hemodynamic system analysis of intraarterial microaxial pumps in vitro and in vivo. Artif Organs 1996;20:650-61.

5. Meyns B, Nishimura Y, Racz R, Jashari R, Flameng W. Organ perfusion with Hemopump device assistance with and without intraaortic balloon pumping. J Thorac Cardiovasc Surg 1997;114:243-53.

6. Spencer FC, Eiseman B, Trinkle JK, Rossi NP. Assisted circulation for cardiac failure following intracardiac surgery with cardiopulmonary bypass. J Thorac Cardiovasc Surg 1965;49:56-73.

7. Jett GK. Abiomed BVS 5000: experience and potential advantages. Ann Thorac Surg 1996;61:301-4.

8. Killen DA, Piehler JM, Borkon AM, Reed WA. Bio-Medicus ventricular assist device for salvage of cardiac patients. Ann Thorac Surg 1991;52:230-5.

9. Pae W. Ventricular assist devices and total artificial hearts: a combined registry. Ann Thorac Surg 1993;55:295-8.

10. Golding LA, Crouch RD, Stewart RW, et al. Postcardiotomy centrifugal mechanical ventricular support. Ann Thorac Surg 1992;54:1059-64. 
11. Noon GP, Ball JW, Short HD. Bio-Medicus centrifugal ventricular support for postcardiotomy cardiac failure: a review of 129 cases. Ann Thorac Surg 1996;61:291-5

12. Miller CA, Pae WE, Pierce WS. Combined registry for the clinical use of mechanical ventricular assist devices: postcardiotomy cardiogenic shock. ASAIO Trans 1990;36:43-6.

13. Golding LAR, Jacobs G, Groves LK, Gill CC, Nose Y, Loop FD. Clinical results of mechanical support of the failing left ventricle. J Thorac Cardiovasc Surg 1982;83:597-601.

14. Golding LAR. Postcardiotomy mechanical support. Semin Thorac Cardiovasc Surg 1991;3:29-32.

15. Mehrige ME, Smalling RW, Cassidy DB, et al. Effect of the Hemopump left ventricular assist device on regional myocardial perfusion and function. Circulation 1989;80(Suppl):III158-66.

16. Laschinger JC, Grossi EA, Cunningham JN, et al. Adjunctive left ventricular unloading during myocardial reperfusion plays a major role in minimizing myocardial infarct size. $\mathrm{J}$ Thorac Cardiiovasc Surg 1985;90:80-5.
17. Sukehiro S, Flameng W. Effects of left ventricular assist for cardiogenic shock on cardiac function and organ blood flow distribution. Ann Thorac Surg 1990;50:374-83.

18. Casimir-Ahn H, Lonn U, Peterzen B. Clinical use of the Hemopump cardiac assist system for circulatory support. Ann Thorac Surg 1995;59:S39-45.

19. Dreyfus GD. Hemopump 31, the sternotomy Hemopump: clinical experience. Ann Thorac Surg 1996;61:323-8.

20. Meyns BP, Sergeant PT, Daenen WJ, Flameng WJ. Left ventricular assistance with the transthoracic 24F Hemopump for recovery of the failing heart. Ann Thorac Surg 1995;60:392-7.

21. Kaplon RJ, Oz MC, Kwiatkowski PA, et al. Miniature axial flow pump for ventricular assistance in children and small adults. $\mathrm{J}$ Thorac Cardiovasc Surg 1996;111:13-8.

22. Butler KC, Maher TR, Borovetz HS, et al. Development of an axial flow blood pump LVAS. ASAIO J 1993;38:M296-300.

23. Siess T, Reul H, Rau G. Concept, realization, and first in vitro testing of an intraarterial microaxial blood pump. Artif Organs 1995;19:644-52.

\section{Timely}

The Journal of Thoracic and Cardiovascular Surgery delivers the information you need now. Articles usually appear within four months of acceptance. 